\title{
SENSOR INTEGRATION FOR ROBOTIC LASER WELDING PROCESSES
}

\author{
Paper 2301
}

\author{
Dimitrios lakovou, Ronald Aarts, Johan Meijer \\ University of Twente, Faculty of Engineering Technology, Laboratory of Mechanical Automation, \\ P.O. Box 217, 7500 AE Enschede, The Netherlands
}

\begin{abstract}
The use of robotic laser welding is increasing among industrial applications, because of its ability to weld objects in three dimensions. Robotic laser welding involves three sub-processes: seam detection and tracking, welding process control, and weld seam inspection. Usually, for each sub-process, a separate sensory system is required. The use of separate sensory systems leads to heavy and bulky tools, in contrast to compact and light sensory systems that are needed to reach sufficient accuracy and accessibility. In the solution presented in this paper all three subprocesses are integrated in one compact multipurpose welding head. This multi-purpose tool is under development and consists of a laser welding head, with integrated sensors for seam detection and inspection, while also carrying interfaces for process control. It can provide the relative position of the tool and the work piece in three-dimensional space. Additionally, it can cope with the occurrence of sharp corners along a three-dimensional weld path, which are difficult to detect and weld with conventional equipment due to measurement errors and robot dynamics. In this paper the process of seam detection will be mainly elaborated.
\end{abstract}

\section{Introduction}

The growth of use of robots for laser welding processes is connected to the increased accessibility in three-dimensional space that robots provide, as well as the possibility to achieve welding velocities as high as $250 \mathrm{~mm} / \mathrm{sec}$. Therefore there is a growing demand for sensory systems that are capable to efficiently guide the laser welding tool over the work piece at high velocities in three-dimensional space. It is essential for these sensory systems to be compact to maintain accessibility, and they should also be able to perform additional functions such as process control and weld inspection.

These requirements introduce the need for integration of various functions in a compact tool. Within this paper a general view will be initially presented on the challenges that robotic laser welding has to face.
Then a description of the developed method, the prototype and its detection capabilities will be outlined. Furthermore, the required sensor calibrations will be explained as well as the sensor processes and the accompanying image processing algorithms. Finally, some experimental results on seam detection will be presented and conclusions will be derived.

\section{Sensing methods overview}

There are a number of methods to implement each of the laser welding functionalities with the use of different types of sensors (tactile, ultrasonic, electromagnetic, etc) [1,2]. Still only few of them can be equally efficient when used for each of the three required sensor functionalities (detection, inspection, process control).

The majority of available sensors make use of optical means (imaging sensors, structured or other type of light) and triangulation methods to obtain the required information [3, 4, 5]. For the three functionalities that are important to laser welding different light sources and imaging means are required.

In general for seam detection, a combination of a imaging sensor (camera) and a projected structured light shape is used. The structured light is projected under an angle in relation to the camera, and therefore triangulation can be used to determine the position of the projected shape. Discontinuities along the structure light shape reveal the position of the weld joint. Uniform light is also required for seam detection at the cases where the structured light is not deformed in any way, for instance the butt joint configuration.

For seam inspection, the same combination of structured light and camera can be used, where detection of discontinuities and deformation of the structured light can provide information about the surface quality of the weld. Additionally, uniform light can also be used to provide images where texture analysis can improve the quality estimation. 
For process control, quite often process light is observed at different wavelengths using photodiodes or even cameras [6].

\section{Laser welding challenges}

Seam detection is the ability to accurately identify the position and orientation of a weld joint in relation to the high power laser tool centre point (TCP). Even though the use of robots increases the accessibility and the capabilities of the welding processes, it also adds additional parameters that need to be taken into account for the seam detection and tracking of the welding process. A number of challenges of robotic laser welding and laser welding in general are outlined next.

\section{Joint configurations}

There are several different joint configurations (Fig.1) that might be required to be welded, each configuration with its own properties. Depending on the properties of each configuration a specific welding approach has to be implemented.

\footnotetext{
Fig.1: Samples of joint configurations from left to right, butt, overlap and corner joint
}

\section{Sharp corners paths}

The appearance of sharp corners along a weld path can influence the seam detection process. Many seam tracking sensors use a structured light source that projects one or more lines on the work piece. These lines are approximately perpendicular to the seam. With such a light source it is very likely that the seam tracking fails after the occurrence of a sharp corner illustrated in Fig.2.
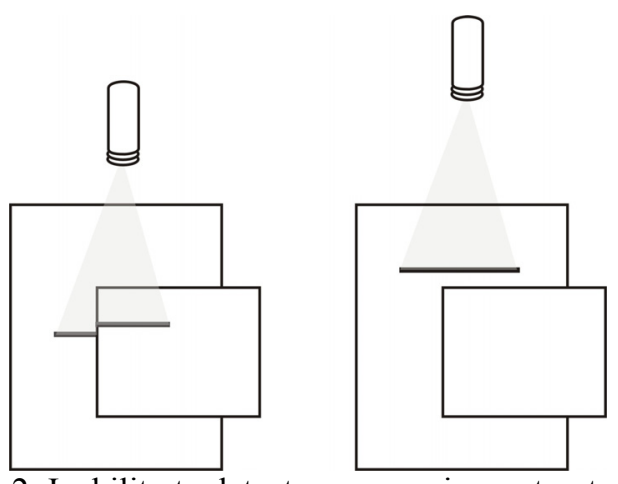

Fig.2: Inability to detect corners using a structured light shape of only a single line

\section{Tool position \& orientation acquisition}

The relative position of the tool towards the work piece in three-dimensional space consists of three positions and three orientations. Most measurement techniques can not acquire all data in one measurement. The number of adjustment measurements that are required for the identification of the complete tool positions and orientations depends on the projected light shape and triangulation method that are used.

\section{Robot dynamics and kinematics}

The majority of the sensory systems are orientation depended. Therefore the sensory system has to keep a certain orientation in relation to the orientation of the weld joint (see e.g. Fig.2). To track and weld such sharp curves and to keep the sensor in the correct orientation, the tool has to perform quick rotations around the high power laser tool axis. In such cases robot dynamics introduce positioning errors that influence the accuracy of the welding process.

\section{Sensor speed}

For seam teaching, the required time for the seam detection is usually not so important, as the joint path can first be taught and then replayed. For real-time seam teaching however, the measurements take place at high processing velocities and the data has to be available in time to correct path errors. That makes the speed of the detection process an important parameter for the robotic implementation of the laser welding processes.

\section{Developed method and prototype}

As already mentioned, most of the problems that appear in a welding process, are related to the physical restrictions and limitations of the sensory system that is used. The developed method attempts to overcome the mentioned sensor limitations and reduce the inaccuracies induced by the robots.

There are two types of information that need to be acquired from this triangulation set-up. The first one involves the measurement of the relative laser tool position and orientation to the work piece, and the second involves the position of the seam and the welding surface quality.

\section{Developed triangulation set-up}

The developed method makes use of the triangulation principles that are applied by most of the existing 
sensory systems. This means that a structured light source projects a shape which is captured by a camera. The axis of the camera and the projection angle of the structured light are under a certain angle.

As it is presented in Fig.3, the developed method makes use of three structured light laser diodes, each projecting a line shape under an angle. The combination of the three structured light lines form a triangular structure light shape similar to the one displayed in Fig.3. Additionally, the three lines are projected in a way that the formed triangular shape is perimetric the high power laser TCP. Furthermore, a mirror is placed along the path of the high power laser beam. This mirror, allows the camera to capture images of the formed triangular shape coaxially to the high power laser.

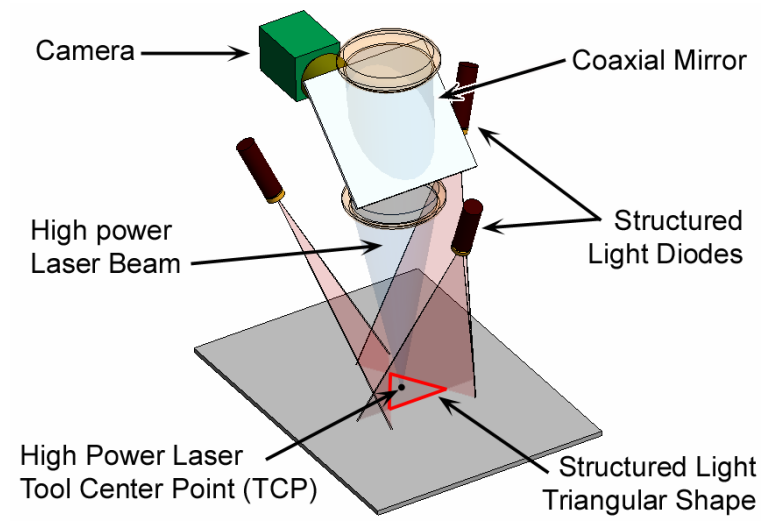

Fig.3: Implemented triangulation scheme

\section{Developed prototype}

The developed prototype is designed as a platform for implementation and testing of the required laser welding functionalities. The prototype is a hexagonal welding head with a coaxial mirror and three holders for the structured light laser diodes (Fig.4).

The six sides of the welding head can be used as mounting bases for installing additional optics, cameras or light sources that are necessary for each of the functionalities. One of the sides is used to attach an interface for the connection with the robot tip.

Inside the welding head there is a rotating mirror holder which is also the mount for the collimator lens. This holder allows the mirror to be rotated towards each of the six sides of the welding head. It can provide a coaxial view to the high power laser to any sensor that is mounted on the side that the mirror faces. The mounting bases and coaxial mirror can also be used by illumination sources or any other system that requires acquiring or projecting coaxially to the high poser laser beam.

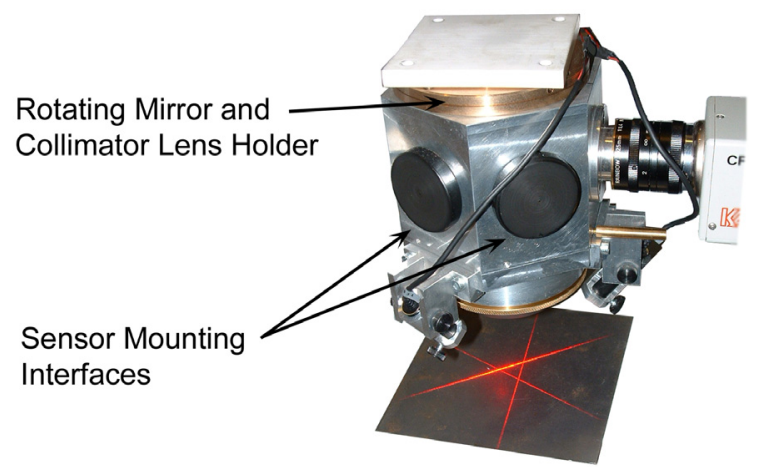

Fig.4: Developed prototype platform with the structured light diodes and camera mounted

The three laser diode holders are used to mount the structured light projectors. Each holder allows the projection of the structured light with a specific angle, as well as the adjustment of the diode's focus lens.

A camera provides pictures of the formed triangle and its characteristics are measured with the use of image processing algorithms.

\section{Relative position and orientation detection}

In three dimensional space, the projected planes of the three diodes form a inverted pyramid with a triangular base (Fig.5.a). Since the view of the camera is coaxial to the high power laser, then the actual axis of the camera is perpendicular to the base of the inverted pyramid. Therefore the diodes have a relative angle to the view of the camera which is the base principle of any triangulation technique. Depending on the position and orientation of the work piece, the projected triangular shape will change size and shape (Fig.5.b, c and d).

The corners of the structured light triangular shape exist on the ribs of the inverted pyramid. Through a sensor calibration procedure (which is explained later in this paper) the projection angles of the triangular shape corner points, and therefore the angle of the pyramid's ribs, are acquired. Therefore by detecting the position of the corners of the triangular shape in two-dimensional space and knowing the relative projection angle, calculation of the three dimensional position of the triangle corner points can be achieved. The calculated three dimensional triangle corner points form a plane which is the plane of the work piece under detection. This plane has a relative 
position and orientation towards the plane of base of the inverted pyramid.

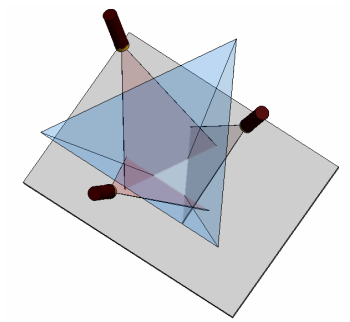

a) Diode formed pyramid

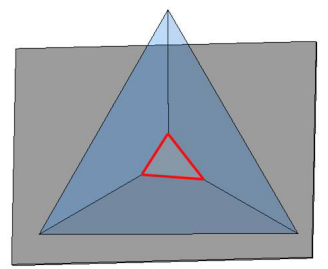

c) Change of orientation

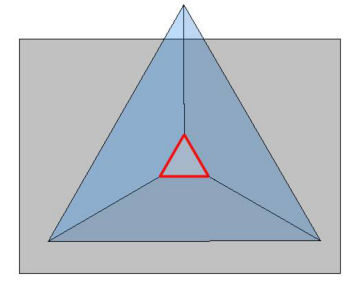

b) Formed triangle

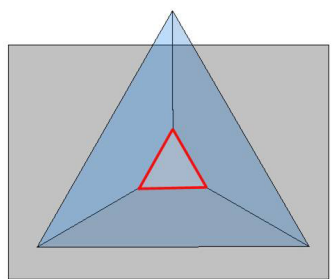

d) Change of position Fig.5: Projected inverted pyramid and changes of triangular shape in relation to work piece position and orientation

In welding processes it is often required to have the tool with different positions and orientations towards the joint path. Therefore it is useful to be able to have relative measurements to the required three dimensional position and orientation. This is implemented by comparing the work piece detected plane's orientation and position with the desired orientation and position that the plane should obtain. In this section the measurement procedure is outlined for a work piece with a flat surface. Therefore, it is necessary to define a "virtual" plane $\mathrm{P}_{\text {Virt }}$ that carries the information about the desired position and orientation. This virtual plane can be expressed with following equation:

$D_{\text {VirtX }} x+D_{\text {VirtY }} y+D_{\text {VirtZ }} z-D_{\text {Virt }}=0$
and its normal vector is defined as: $\underline{n}_{\text {Virt }}=\left[\begin{array}{c}D_{\text {VirtX }} \\ D_{\text {VirtY }} \\ D_{\text {VirtZ }}\end{array}\right]$

The welding head's camera is continuously capturing images of the projection of the structured light on the work piece. For each image, the triangle corners points are acquired and their three-dimensional coordinates calculated. The plane that is formed from the triangle corner points is defined as $\mathrm{P}_{\mathrm{New}}$ and its normal vector is $\underline{n}_{\text {New }}$. As already explained previously, the $\mathrm{P}_{\mathrm{New}}$ is actually the plane of the work piece.
To determine the orientation and position of a work piece in relation to the welding head, the dihedral angle between every new plane and any user defined 'virtual' plane has to be calculated. The dihedral angle between the two planes is calculated via the dot product of the plane normal vectors.

In Fig.6, the angle that is formed between two surfaces is displayed. Additionally, the normal vectors $\underline{n}_{\text {Virt }}$ and $\underline{n}_{\text {New }}$ of the virtual and "new" work piece planes are also displayed. As was mentioned before the angle between the normal vectors is equal to the angle between the planes. Still the problem that remains is the axis around which the new plane should be rotated to become parallel to the calibration plane.

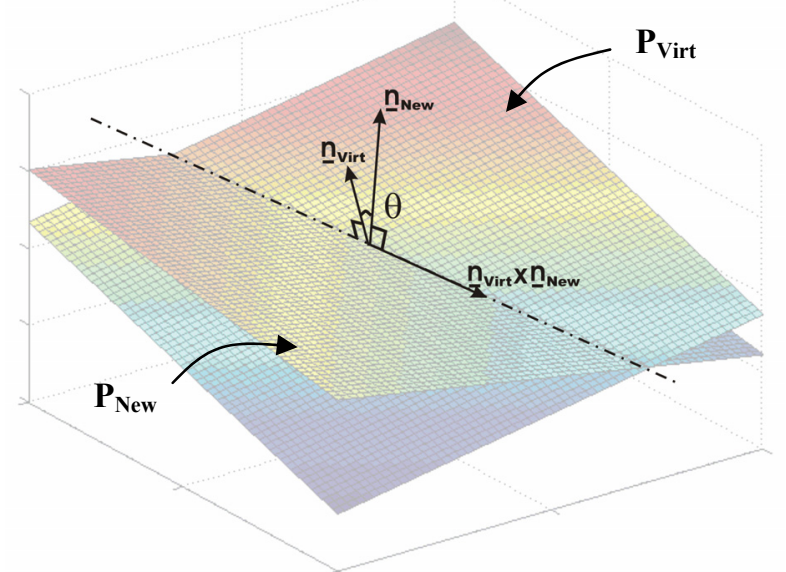

Fig.6: Normal vectors \& Rotation axis

When the two normal vectors are set on a common starting point, they form a plane. The dihedral angle " $\theta$ " belongs to that plane. Therefore, it is required to rotate the image plane around an axis which is perpendicular to the plane of the normal vectors. This axis can be derived from the cross product of the two normal vectors $\underline{n}_{V i r t} \times \underline{n}_{\text {New }}$ as shown on Fig.6. This axis is also parallel to the line that is formed from the intersection of the two image planes. The origin of this rotation axis is placed at the point where the zaxis of the sensor coordinate system penetrates the virtual plane.

\section{Seam detection}

For the seam detection process it is necessary to have prior information about the configuration of the weld joint. As it is displayed in Fig.7, different joint configurations create different effects on the structured shape. Therefore, information about the type of joint configuration in relation to the detected 
deformations and discontinuities along the boundaries of the structured light shape can provide information about the position of the weld joint.
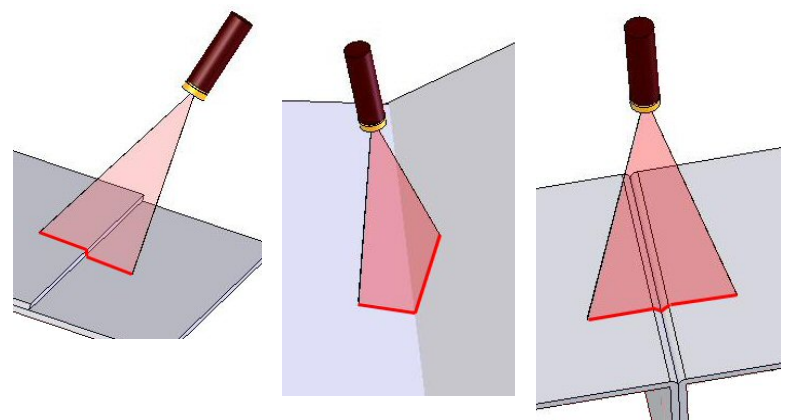

Fig.7: Deformations of structured light lines due to different joint configurations

For the developed set-up, the influence of the different joint configurations is displayed in Fig.8. For each case different detection algorithms need to be used. For instance, in the case of overlap joints, discontinuities appear along the structured light lines, whereas in the case of butt joints, nothing changes along the structured light shape and therefore other ways of detection need to be used.

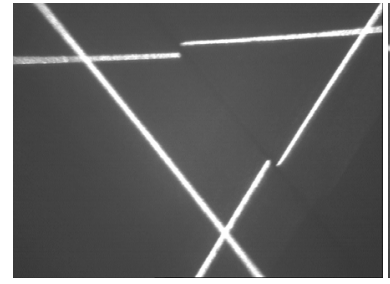

a) Overlap joint

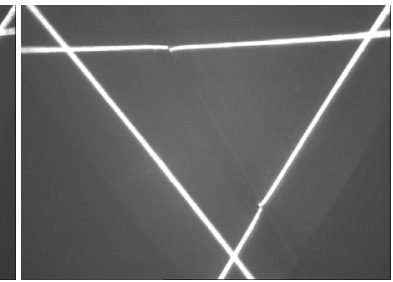

b) Butt joint

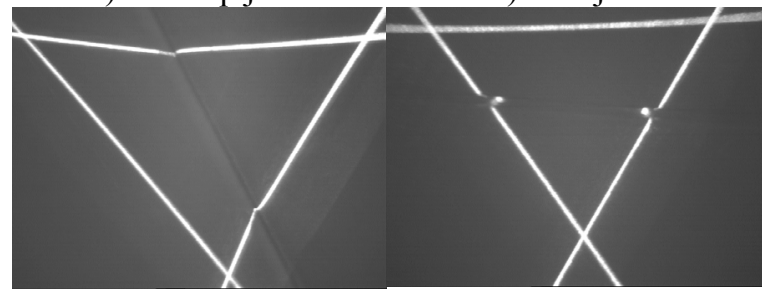

c) Corner Joint

e) Concave butt joint

Fig.8: Examples of projected structured light on overlap joint (left) and butt joint (right)

\section{Seam inspection}

The same set-up is also used for seam inspection. The detection principle remains the same as the detection method depends on the discontinuities or deformations of the structured light lines. As for seam detection, prior knowledge of the joint configuration is required for the inspection process.

Fig.9 presents examples of the deformations that the welded joints introduce on the structured light shape.
The detection of the surface welded quality is performed in a region of interest around the deformed structured light shape.

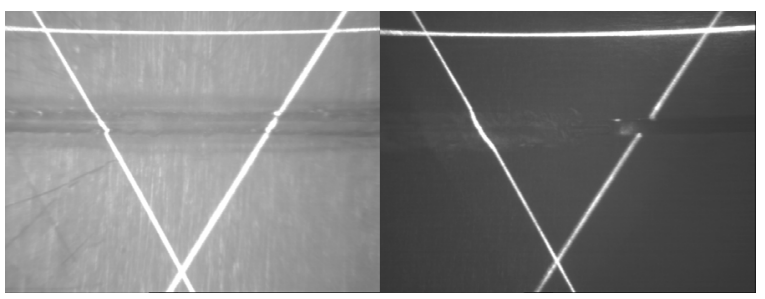

a) Only Inspection

b) Detection and Inspection

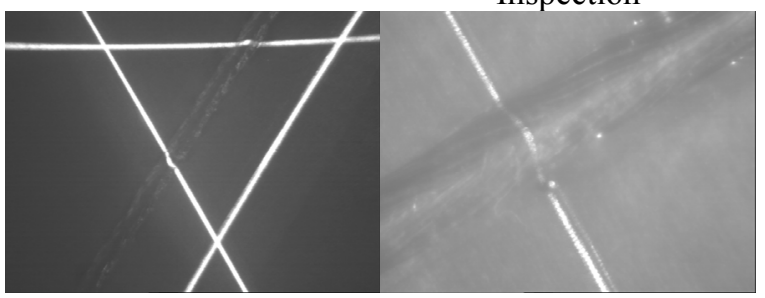

c) Under an angle

e) Region of interest

Fig.9: Deformations of the structured light due to the quality of the seam

\section{System calibration}

It is common that different high power laser focus lengths are used on the same welding system, according to the welding process that needs to be implemented. A change of the focus lens of the high power laser beam, results to the altering of a number of parameters of the sensor that need to be estimated and recalibrated. For instance the projection angle of the structured light diodes needs to be changed in order to maintain the perimetric projection around the laser TCP. Furthermore, in order to keep the same camera field of view around the laser TCP, the camera optics also needs to be changed. Any change in the configuration of the optical path of the welding head results to different magnification and different lens distortions that need to be identified.

\section{Lens calibration}

The identification of the lens distortions introduced by the lenses along the optical path is implemented with lens calibration.

The focussing lens for the high power laser is not optimised to generate distortion free images with large field of view. This could result to distortions especially near the borders of the image (Fig.10 left). For accurate measurements it is necessary to be able to identify these distortions and use the information to undistort the image. 
Several lens calibration techniques are available in literature. The main approach used is by identifying the distortions appearing in a known pattern. In Fig.10 the original image of the triangular shape is compared with an undistorted one that is the result of the calibration method found in [7].

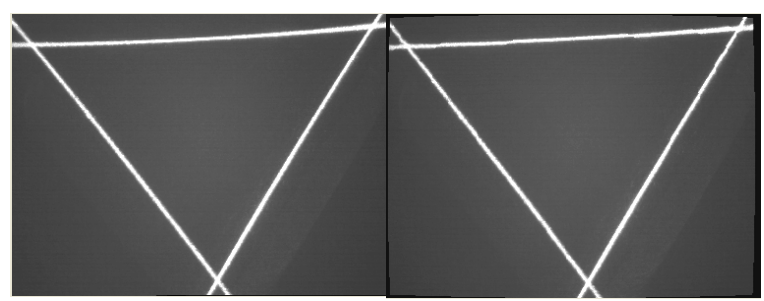

Fig.10: On the left the origial image where visibly the lines are distorted, on the right the undistorted image

Each time one of the lenses of the optical system (high power laser focussing lens and/or camera optics) is changed, a lens recalibration is necessary. The recalibration is required to assure that correctly undistorted images are used for any other calibration or measurements that are to be carried out next.

\section{Sensor calibration}

To maintain the perimetric feature of the projected triangle around the laser TCP, it is necessary to be able to change the projection angle of the structured light diodes when the laser focus lens is changed. The new projection angles will produce a different inverted pyramid whose parameters must be estimated.

The purpose of the sensor calibration is to obtain the parameters of the inverted pyramid and the mathematical expressions that relate features in the camera images with actual three-dimensional positions and orientations. The input of the calibration consists of images with triangles as in Fig.10 that are observed when a perfectly flat surface is positioned underneath the welding head.

The sensor calibration process requires that the projected triangles corners can be detected. This task is implemented by applying image processing algorithms on the images that are captured by the camera. An additional requirement of the sensor calibration process is to have the optical axis of the high power laser perpendicular to a flat surface. It is assumed that this can be achieved somehow. Also the exact position of the laser TCP must be known.

The sensor calibration method is based on the inverted pyramid property of the projected structured light. Since the structured light lines form an inverted pyramid, there must be one position in threedimensional space where the ribs of the pyramid converge (tip of the inverted pyramid). This point of convergence of the pyramid is the sensor's centre point (SCP).

For the calibration a series of images is acquired while the robot positions the tool at a number of positions. By displacing the tool along the optical axis a different size of the same structured shape is projected on the flat surface. By acquiring the coordinates of the corners of the shape and combining it with the relative displacement of the tool, a number of three dimensional corner coordinate data is obtained. Then a line fitting implementation on the data is performed. This results to the equations of the inverted pyramid's ribs that the corners points exist on, in three-dimensional space (Fig.11)

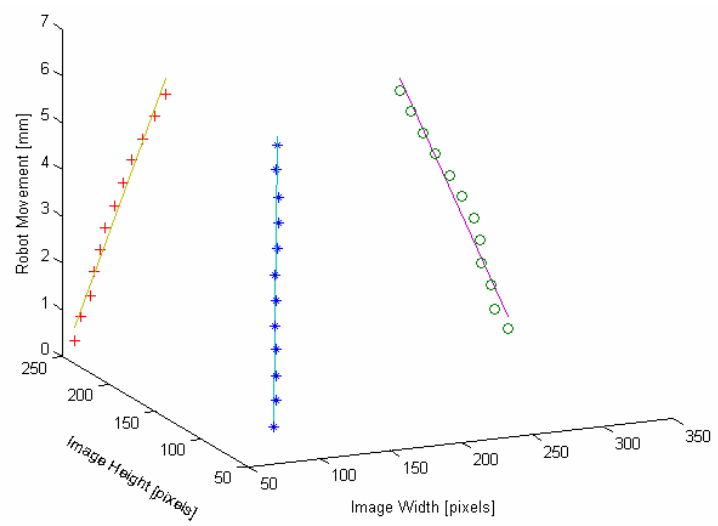

Fig.11: Calibration data of the triangular shape corner points and fitted pyramid ribs

From the acquired pyramid rib equations, the point of the tip of the inverted pyramid in three-dimensional space can be calculated.

By knowing the exact position of the laser TCP, the relation with the SCP can be derived. Thus, sensor measurements can be related directly to the laser tool.

\section{Image processing}

\section{Introduction}

The main task of image processing is the identification of the lines or parts of lines that form the triangular shape in the captured images. From the identified lines, the corners of the triangular shape can be calculated. Once the triangle corner points have been identified the relative position and orientation of the tool to the work piece and the seam can be calculated as was explained in 'Relative position and orientation detection' paragraph. 
For the correct interpretation of the line detection results, prior knowledge of the joint configuration under detection is also required. For instance, in image Fig.8.a the seam position can be detected from the discontinuities along the structured light shape, whereas in image Fig.8.c the seam can be detected from the bending parts of the lines.

The accuracy of the image processing measurements depends on the resolution of the imaging sensor and the accuracy of the processing algorithms. Image processing is a time intensive process, therefore a greater accuracy usually requires more time. For this reason, the obtained accuracy may be reduced when high measurement frequency is needed

\section{Image processing algorithms}

In Fig.12 some of the main image processing steps for the detection of the lines and the acquisition of their junction points are being displayed.

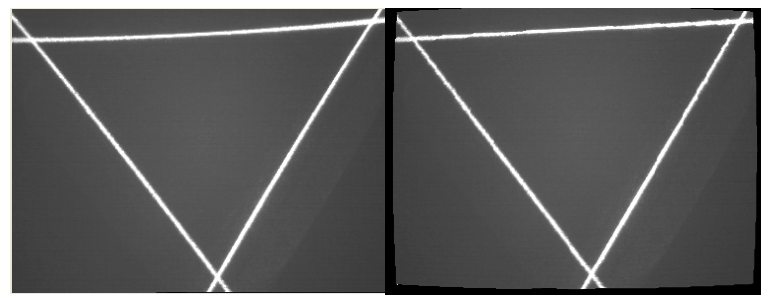

a) Original Image

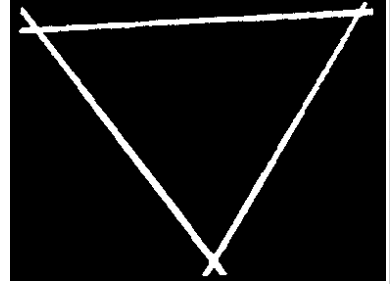

c) Remove noise b) Undistort

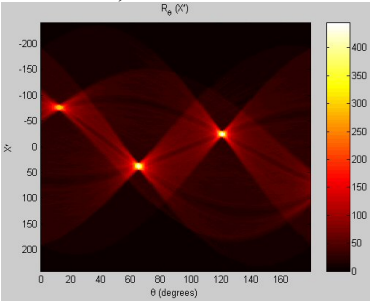

d) Line detection

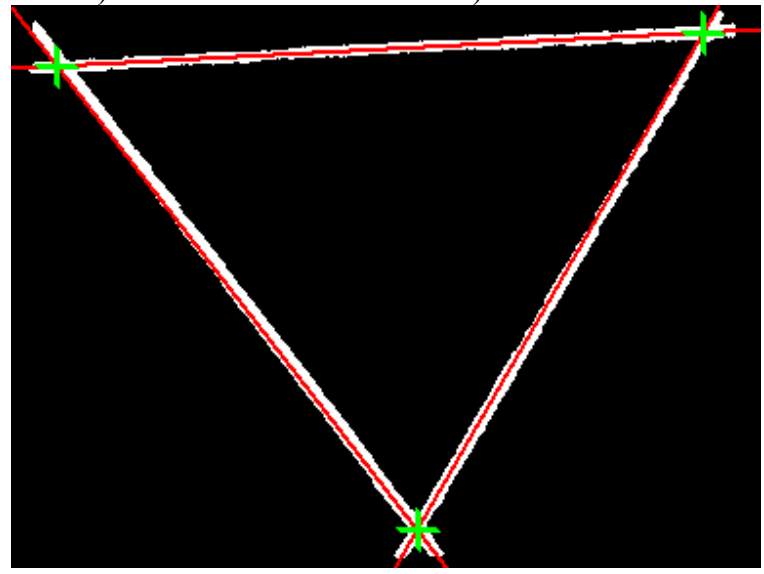

e) Final result
There are several techniques that can be used to detect multiple lines in a single image. Some of them require dedicated hardware configurations and some of them can just involve a software implementation. At the current development stage a more generic line detection technique is being used with the help of a software implementation of a Radon [8] transform. Several other methods are also under investigation for efficiency comparison to the Radon algorithm.

Once the image (Fig.12.a) has been undistorted (Fig.12.b), cleared from noise and the triangular shape has been clarified (Fig.12.c), the Radon algorithm is applied on the image. The radon transform draws a two-dimensional map of all the lines that can pass through a certain pixel of the original image. Each line is represented on the radon map (Fig.12.d), according to its distance from the original image centre and its orientation. Therefore the lines that are pasting through more pixels have higher illumination values on the radon map. A local maxima algorithm implementation on the Radon map will result with the number of the detected lines and their position and orientation in the real image.

\section{Experimental results}

\section{General Overview}

In Fig.13 en example of a seam detection output image from the developed sensory system is displayed. At this example the seam detection has been performed on overlap joint configuration. As expected, discontinuities along the structured light shape are evident. The separate parts of the triangular shape have been labelled and displayed in Fig.13 with different grey values (white, and dark grey)

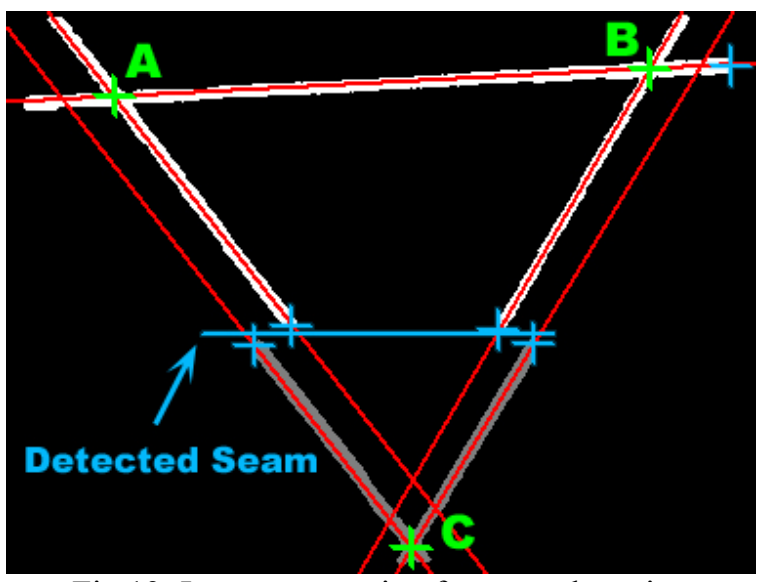

Fig.13: Image processing for seam detection

Fig.12: Image processing process 
The detected lines of the triangular shape are also displayed along with their junctions on the corner points $\mathrm{A}, \mathrm{B}$ and $\mathrm{C}$. These are the points that will be used for the identification of the orientation and position of the welding head in relation to the work piece.

A line with the position of the detected seam is also displayed. To determine the parameters of this line the position of the structured light discontinuities must first be determined (displayed with crosses on both sides of the detected seam line). Then, a linear square fit of the discontinuity points can provide with the line of the detected seam.

At Fig.13, the process of seam detection is presented without performing any laser welding operations. In the case where seam detection, welding and inspection is being performed simultaneously, one of the brake points would exist at the area where seam inspection is being performed as the joint will already have been welded.

\section{Orientation free overlap joint detection}

As was explained in "Laser welding challenges" paragraph, the requirement to keep a certain sensor orientation relative to the seam can introduce problems due to robot dynamics. In Fig.14, examples of seam detection cases are displayed regardless the sensor-seam relative orientation.
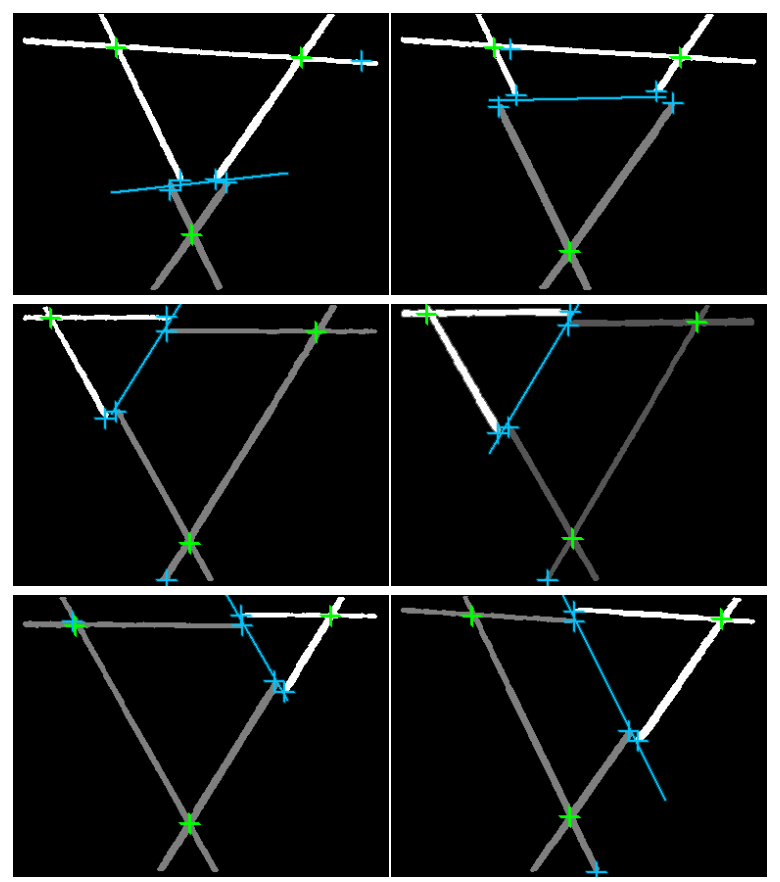

Fig.14: Examples of overlap joint configuration seam detection regardles the seam position and orientation
The joint configuration in Fig.14 image series is always overlap joint. The first two images of Fig.14 present results of the seam detection image processing algorithm. Each pair of images displays a certain orientation of the seam crossing the triangular shape from different positions (lower part, left right). The line (blue) that separates the two parts of the triangular shape is the detected seam.

\section{Sharp corner detection of overlap joints}

Another challenge that the seam detection sensors face is the existence of sharp corners. In Fig.15 a series of images are presented that display the detection of sharp corners along a seam. For this image series a sharp corner was places under the sensor and was asked to be detected.

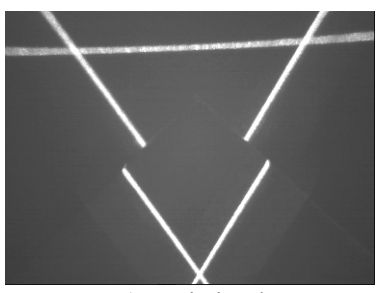

a) Original

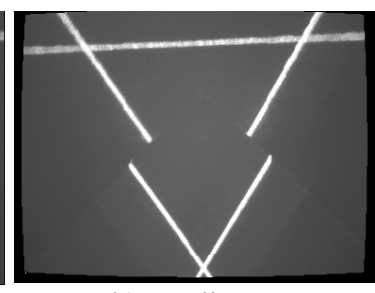

b) Undistort

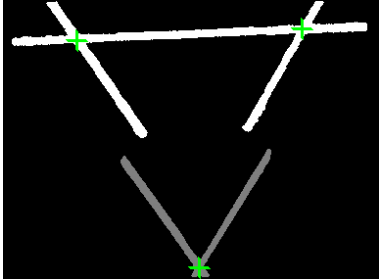

c)Corner points

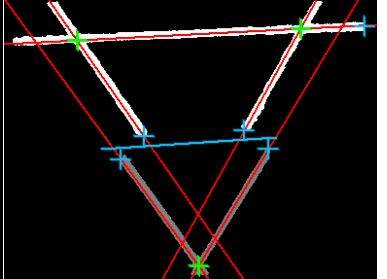

d) Seam \& Corner lines
Fig.15: Examples of corner joint configuration detection

As it can be seen from the images Fig.15.c and Fig.15.d, the seam detection process of the sharp corners does not differ much from the normal overlap joint case when approached from a static point of view. The identification of the shape of the corner can only be resulted from a seam tracking process, where interpolation of the detected seam points takes place.

\section{Conclusions}

The developed prototype is capable of seam detection of overlapped joint configurations regardless the seam orientation position or shape. It can extract all the required three-dimensional seam parameters from every single captured image. Still, further optimisations are necessary to speed up the time intensive image processing process. 


\section{Acknowledgements}

This work is financially supported by the Dutch Technology Foundation STW under grant TWO.5927. Furthermore, the authors would like to acknowledge the work of Leo Tiemersma and Jorg Entzinger from the University of Twente and their valuable input in the progress of this project.

\section{Bibliography}

[1] Camplin, K.R. Laser weld Inspection with electro-magnetic acoustic transducers (EMATs): Innerspec Technologies Inc, Virginia USA.

[2] Bates, G., Kelkar G. Determination of weld quality in pulsed Nd:YAG laser welds using IR and acoustic sensors: Unitek Miyachi International, California USA.

[3] Jackel, T., Miesner, J., Frischkorn, F., Kreitlow, H., Jackson, M.R., Parkin, R.M. (2003) Laser Welding seam tracking system: ICOM 2003 London, Professional Engineering Publishing.

[4] Bosner, G., Parker, G.A. (1999) Robotic gas metal arc welding of small diameter saddle type joints using multistripe structured light: Society of Photo-Optical Instrumentation Engineers.

[5] Arakawa, K., Kakizaki, T., Omyo, S. (2000) A machine vision approach to seam sensing for highspeed robotic sealing, IEICE TRANS. INF \& SYST., Vol E83-D, No\#7.

[6] Postma, S. (2003) Weld Pool Control in Nd:YAG Laser Welding: 90-77172-05-X, University of Twente.

[7] Zhang, Z. (2002) A flexible new technique for camera calibration: Technical report MSR-TR-98-71: Microsoft Research, Microsoft Corporation, Redmond.

[8] Toft, P. (1996) The Radon Transform - Theory and Implementation, PhD Thesis, IMM, DTU. 